\title{
The Psychological Impact of the COVID-19 Pandemic on Egyptian Diabetic Patients
}

\author{
Inass Shaltout ${ }^{1}$, Khaled Ahmed Attia ${ }^{2}$, Alia Saleh², Hassan Shora ${ }^{3}$, and Mazen Ahmed \\ Attia $^{2}$ \\ ${ }^{1}$ Affiliation not available \\ ${ }^{2}$ Cairo University Kasr Alainy Faculty of Medicine \\ ${ }^{3}$ Port Said University
}

October 11, 2020

\begin{abstract}
Background: Since the onset of the COVID-19 pandemic, concerns about health-related and economic hazards of the virus, the restrictions imposed by the public health measures, the lack of social interactions, and the financial losses have affected people's mental health in various ways. People with diabetes are more vulnerable to comorbid mental illness that may interfere with treatment adherence and disease prognosis. This study explores the psychological state of Egyptian patients suffering from diabetes mellitus (DM) during the COVID-19 pandemic. Methods: Egyptian adults with DM were invited to participate through an online survey in the period from April 24 to May 12. The survey included sociodemographic information, questions assessing diabetes status, and general assessment of the psychological health status using the General Health Questionnaire (GHQ-12). Results: In total, 849 participants shared in the survey. $51.4 \%$ of the studied population were at risk, while $21.2 \%$ were at high risk of psychological distress. There was a statistically significant difference with the non-working group with a mean GHQ-12 score of $17.44, \mathrm{SD}=6.267, \mathrm{SE} 0.491,95 \%$ (16.47-18.41), and p-value of 0.01 . Regarding the disease precautions, there was a statistically significant difference between home isolation and those who did not home isolate with a mean 16.23 and SD 6.285 compared to a mean 15.32 SD 5.701 p-value 0.028 . There was a significantly increased number of 4 or more diabetes complications and comorbidities with a mean 20.60 SD 8.675, SE 2.240, (95\% CI 15.80-25.40) with a p-value of less than 0.001 and mainly with hypertension as comorbidity. Conclusion: The current study shows that Egyptian patients suffering from DM are likely to psychological distress amid the COVID-19 pandemic. Risk factors included female gender, non-working, and patients with diabetic complications, particularly hypertension. Further attention to the psychological needs of Egyptian patients with DM amid the COVID-19 is recommended.
\end{abstract}

\section{Hosted file}

Pandemic COVID - DM (Final).pdf available at https://authorea.com/users/366255/articles/ 486039-the-psychological-impact-of-the-covid-19-pandemic-on-egyptian-diabetic-patients 